\title{
Determinación de volúmenes pulmonares mediante pletismografía en pacientes con fibrosis quística
}

\author{
ILSE CONTRERAS E.*, SOLANGE CAUSSADE L.*, LUIS VEGA B.*, GISELA MOYA D.*, \\ CLAUDIO CALLEJAS C.**, CONSUELO ARANGUIZ G.** e IGNACIO SÁNCHEZ D.*
}

Lung volume determinations by whole plethysmography in cystic fibrosis patients: A preliminary report

Introduction: Cystic fibrosis (CF) patients develop a progressive impairment of pulmonary function tests (PFT) by recurrent infection. Objective: To evaluate PFT in CF patients using spirometry, oxygen saturometry $\left(\mathrm{SaO}_{2}\right)$ and whole plethysmography (WP) for determining possible mathematical relationships. Methods: FVC, FRC, RV, TLC, FEV,$F E F_{25-75}$, obtained in 10 CF patients were compared with Zapletal's reference values and with the values obtained in 33 healthyschool children. Results: Mean age 13.6 years-old (range: 9-20). All CF patients had normal $\mathrm{SaO}_{2}$, $F E V_{1}$ was normal in five (four had FEV $V_{1}$ between 65-80\%), 4 had RV/TLC > 30\%, 3 had increased FRC and RV/TLC, only one CF patient had WP normal. The average of TLC and RV were higher than reference values and control group $(p<0.05)$. We found an inverse relationship between $F E V_{1}$, $F_{25} F_{25}$ and RV/TLC $[r=-0.642(p<0.05)$ and $r=-0.803(p<0.01)$; respectively]. Conclusion: This study suggests an increase of pulmonary volume in CF patients with not necessarily abnormal spirometry and an inverse mathematical relationship between $F E V_{1}, F E F_{25-75}$ and RV/TLC.

Key words: Whole plethysmography, lung function, cystic fibrosis, children.

\section{Resumen}

Introducción: La fibrosis quística (FQ) exhibe un deterioro progresivo de la función pulmonar (FP) asociada a infecciones recurrentes. Objetivo: Evaluar la FP en sujetos con FQ mediante espirometría, saturometría y pletismografía (PG) determinando alguna relación matemática. Pacientes y Métodos: Se identificaron 10 pacientes capaces de realizar CVF, CRF, VR, CPT, VEF , y $\mathrm{FEF}_{25-75 \%}$; comparándose con los valores de Zapletal y de 33 controles sanos. Resultados: Edad promedio 13,6 años (rango: 9-20). $\mathrm{SaO}_{2}$ y $\mathrm{VEF}_{1}$ fueron normales en 10 y 5 sujetos, respectivamente (4 tenían VEF $\mathrm{F}_{1}$ entre 65-80\%). La PG mostró 4 pacientes con VR/CPT > 30\%, 3 con aumento de CRF y VR/CPT, sólo uno tuvo PG normal. Los promedios de CPT y VR fueron superiores a valores de referencia y controles $(p<0,05)$. Se encontró una relación inversa entre $V E F_{1}$ y $V R / C P T(r=-$ 0,642, $p<0,05)$ y entre $F E F_{25-75}$ y VR/CPT $(r=-0,803, p<0,01)$. Conclusiones: Este estudio sugiere un incremento significativo de los volúmenes pulmonares en pacientes con FQ aún teniendo espirometría normal; así como una relación matemática inversa entre $V E F_{1}, F_{25} F_{25-75}$ y VR/CPT.

Palabras clave: Pletismografía, función pulmonar, fibrosis quística, niños.

\footnotetext{
* Sección Respiratorio Pediátrico. Escuela de Medicina. Departamento de Pediatría. Pontificia Universidad Católica de Chile.

** Interno(a) de Medicina. Escuela de Medicina. Pontificia Universidad Católica de Chile.
} 


\section{Introducción}

La progresión de la enfermedad respiratoria, determinada por la pérdida de la función pulmonar (FP), es la causa más frecuente de morbimortalidad en los pacientes con fibrosis quística $(\mathrm{FQ})^{1-2}$. La velocidad de esta progresión, determina la severidad del compromiso respiratorio en niños, por lo que diversos esfuerzos están orientados a la detección precoz y control de este deterioro funcional ${ }^{1-3}$.

Las pruebas de función pulmonar (PFP) tienen un rol importante en determinar la evolución, manejo y control de los pacientes con FQ. Estas incluyen: $\mathrm{SaO}_{2}$, espirometría, lavado de nitrógeno, dilución de helio y pletismografía entre otras ${ }^{4,5}$. Muchas de las conductas terapéuticas (uso de broncodilatadores, antibióticos, etc.) son controladas mediante espirometrías bimensuales o trimestrales, determinándose así la respuesta a estas terapias. Si bien el $\mathrm{VEF}_{1}$ ha sido la variable más utilizada para determinar la evolución y progresión de estos pacientes ${ }^{3,6}$; existen autores que sugieren que su deterioro o alteración podría no ser precoz. Dependiendo de la edad del paciente, las PFP se convierten en un desafío cada vez más complejo por lograr mediciones precoces y confiables. Otra manera de determinar el compromiso pulmonar es a través de la radiografía de tórax y la utilización de puntajes radiológicos ${ }^{7}$. Es así, como en los últimos años, el avance en el campo de la imagenología le ha otorgado un rol importante a la tomografía computarizada de alta resolución (HRCT) en el diagnóstico precoz de las alteraciones de la vía aérea ${ }^{8}$.

La pletismografía (PG) permite determinar los volúmenes y capacidades pulmonares en sujetos con condiciones agudas y crónicas ${ }^{4,9,10}$. Determinar el VR, CRF y CPT permite identificar el compromiso obstructivo en forma más precoz ${ }^{5,6}$. Por otra parte, la PG es considerada como el examen de elección para la determinación temprana de la hiperinsuflación y atrapamiento aé$\mathrm{reo}^{4}$. Algunos grupos han mostrado el rol de la PG en algunas condiciones crónicas respiratorias como: asma, displasia broncopulmonar y $\mathrm{FQ}^{8-11}$; sin embargo, no existen estudios en nuestro medio que evalúen esta experiencia. Nuestro objetivo fue determinar los valores de volúmenes pulmonares (CRF, VR, CPT y la relación $\mathrm{VR} / \mathrm{CPT}$ ) en un grupo de pacientes con FQ conocidos y controlados en nuestro centro, evaluando la relación matemática con flujos y volúmenes pulmonares, así como con la condición clínica del paciente, con la intención de buscar la forma de evaluar y determinar qué tipo de compromiso pulmonar es más precoz en este tipo de pacientes.

\section{Pacientes y Métodos}

De manera retrospectiva, durante el período 2003-2004, se identificó a los 32 pacientes con FQ controlados en el Laboratorio de Respiratorio Pediátrico de nuestro centro, identificando a 10/32 sujetos mayores de 6 años, capaces de realizar maniobras pletismográficas adecuadas (edades entre 9 y 20 años, 7 de sexo masculino) según los criterios y recomendaciones de la ERS/ATS ${ }^{12}$. En breve: se le solicita al paciente que se siente en el interior de una cabina herméticamente cerrada; posteriormente se le indica que respire por la boca a través de una boquilla con una pinza nasal, realizando una maniobra de "jadeo" tranquilo por 3 ciclos, para adaptación a la boquilla del pletismógrafo, después el explorador cierra la válvula durante unos segundos, (por lo tanto la entrada de aire al sistema), solicitándole al sujeto que continúe con los esfuerzos respiratorios para vencer la resistencia. Una vez abierta la válvula, el sujeto realiza inmediatamente una espiración forzada seguida de una inspiración forzada hasta CPT y de nuevo una maniobra espiratoria forzada para después respirar tranquilamente y terminar la prueba.

En todos los casos se solicitó autorización a los padres para la participación de sus hijos; realizando a cada uno de ellos, por un mismo operador, espirometría y PG (tres maniobras aceptables y reproducibles según criterios ATS); $\mathrm{SaO}_{2}$ y radiografía de tórax. Se aplicó un cuestionario para registrar datos generales, síntomas, índice masa corporal (IMC) [(peso $(\mathrm{kg}) /$ talla $(\mathrm{m})^{2}$ ], colonización bacteriana y tratamiento, al momento de la PG. El puntaje de gravedad de FQ se determinó según la escala de Brasfield (radiología) $^{7}$ y Shwachman (clínica) ${ }^{13}$.

Se midió $\mathrm{CVF}, \mathrm{VEF}_{1}, \mathrm{FEF}_{25-75 \%}$, la relación $\mathrm{VEF}_{1} / \mathrm{CVF}$, VR, CPT, CRF y la relación VR/ CPT. Todas las pruebas de FP, se realizaron durante un período de estabilidad clínica, determinado por el médico tratante, empleando un pletismógrafo con espirómetro incorporado Medgraphics Breeze Modelo 1070-1085 (St. Paul, Minnesota, USA) y un oxímetro de pulso Omheda Modelo Biox 3700 (Boulder, CO, USA). Se emplearon como valores de referencia los publicados por Knudson y Zapletal ${ }^{14-15}$ y se compararon los volúmenes pulmonares de estos pacientes con un grupo control de 33 sujetos vo- 
luntarios sanos chilenos, pareados por sexo y talla obtenidos de un estudio paralelo de determinación de valores normales de PG en el Laboratorio Respiratorio Pediátrico de nuestra institución ${ }^{16}$.

Para efectos de este estudio se definió como una $\mathrm{SaO}_{2}$ normal aquella $\geq 94 \%$; obstrucción bronquial como $\mathrm{VEF}_{1}$ o $\mathrm{VEF}_{1} / \mathrm{CVF}<80 \%$, $\mathrm{FEF}_{25-75 \%}<60 \%$; atrapamiento aéreo como VR/ CPT $>30 \%$ con CPT normal o VR $>130 \%$ e hiperinsuflación pulmonar como CPT > 130\%. Por tratarse de un estudio piloto con un número de pacientes reducido, no se determinó cálculo muestral teórico necesario. Para el análisis estadístico se utilizó las pruebas de Mann-Whitney y KrusKal-Wallis considerando como significativo $\mathrm{p} \leq 0,05$. Este estudio contó con la aprobación del Comité de Ética de nuestra institución.

\section{Resultados}

La edad promedio de los pacientes fue 13,6 años (rango: 9-20 años) con una edad de diagnóstico promedio 2,2 años (rango: 22 días-7 años), de los cuales 1 fue en el período de recién nacido por el antecedente de un hermano con FQ, 7/10 eran varones, presentando 9/10 un estado nutricional en rango de normalidad. La mutación $\Delta$ F508 fue la más frecuente identificada (6/10). En 7/10 se demostró colonización bacteriana con $P$. aeruginosa o $S$. aureus; los 7 colonizados presentaban además bronquiectasias en el TAC de tórax; 2 presentaron flora habitual y un paciente no tenía ningún microorganismo aislado. Los puntajes radiológicos revelaron que todos se encontraban en estado leve-moderado de enfermedad (Tabla 1). El grupo control, tenía una edad promedio de 12,4 años (rango: 915 años) siendo 21 varones, todos eutróficos y sin antecedentes mórbidos previos.

Los estudios de $\mathrm{SaO}_{2}$ fueron normales en todos los casos, existiendo sólo un paciente con las tres PFP normales. Sin embargo, en 9/10 niños se encontró algún grado de alteración en la PG (VR promedio: 323\%, rango: 135-551), de ellos, 4 presentaron $\mathrm{VEF}_{1}$ normal y 2 tuvieron espirometría totalmente normal. El $\mathrm{VEF}_{1}$ fue $>$ $80 \%$ del teórico para la edad en 5 sujetos, entre $65-80 \%$ en 4 y sólo uno mostró un patrón restrictivo. De los 5 casos con $\mathrm{VEF}_{1}$ normal, 2 tenían $\mathrm{FEF}_{25-75 \%}$ bajo el valor normal $(<70 \%)$. En 7/10 pacientes se observó un $\mathrm{FEF}_{25-75}$ disminuido menor a $60 \%$. En $3 / 10$ se observó respuesta (cambio mayor o igual al $10 \%$ del valor basal de $\mathrm{VEF}_{1}$ ) luego de la administración de salbutamol; uno de ellos tenía una CRF $>160 \%$. Ningún paciente presentó respuesta paradojal al broncodilatador. En la PG, en 7 casos se encontró CRF aumentada (mediana: 143\%, rango: 106-315); 2 tuvieron hiperinsuflación pulmonar (mediana: 182\%, rango: 147-216), todos con

Tabla 1. Características clínicas y de laboratorio en 10 pacientes con FQ

\begin{tabular}{|c|c|c|c|c|c|c|c|c|c|c|c|c|c|c|}
\hline $\begin{array}{l}\text { Edad } \\
\text { (años) }\end{array}$ & Sexo & IMC & IMCр & $\begin{array}{c}\text { Tos } \\
\text { crónica }\end{array}$ & $\begin{array}{l}\text { Microorga- } \\
\text { nismo } \\
\text { identificado }\end{array}$ & $\begin{array}{c}\text { Bronquiec- } \\
\text { tasia en } \\
\text { TAC }\end{array}$ & $\begin{array}{c}\text { Puntaje } \\
\text { de } \\
\text { Schwachman }\end{array}$ & $\begin{array}{c}\text { Puntaje } \\
\text { de } \\
\text { Brasfield }\end{array}$ & $\begin{array}{c}\mathrm{SaO}_{2} \\
(\%)\end{array}$ & $\begin{array}{c}\% \\
\mathrm{VEF}_{1}\end{array}$ & $\begin{array}{c}\% \\
\mathrm{FEF}_{25-7}\end{array}$ & $\begin{array}{c}\% \\
\text { CPT }\end{array}$ & $\begin{array}{l}\% \\
\text { VR }\end{array}$ & $\begin{array}{l}\mathrm{VR} / \\
\mathrm{CPT}\end{array}$ \\
\hline 9 & F & 17,4 & 70 & $\mathrm{Si}$ & P. aeruginosa & $\mathrm{Si}$ & 70 & 22 & 95-96 & 97 & 90 & 109 & 142 & 34 \\
\hline 10 & F & 16,8 & 50 & $\mathrm{Si}$ & S. aureus & $\mathrm{Si}$ & 70 & 22 & 96-97 & 65 & 118 & 118 & 257 & 56 \\
\hline 11 & M & 16,6 & 25 & $\mathrm{Si}$ & Flora habitual & No & 65 & 23 & 95-98 & 83 & 58 & 101 & 135 & 32 \\
\hline 11 & M & 17,1 & 50 & No & no & No & 70 & 23 & 94-95 & 108 & 147 & 147 & 271 & 43 \\
\hline 13 & M & 21,7 & 80 & $\mathrm{Si}$ & P. aeruginosa & $\mathrm{Si}$ & 65 & 21 & 94-98 & 88 & 44 & 216 & 551 & 61 \\
\hline 14 & F & 19,8 & 55 & $\mathrm{Si}$ & S. aureus & $\mathrm{Si}$ & 65 & 20 & 94-95 & 77 & 51 & 114 & 243 & 43 \\
\hline 14 & M & 15,4 & -5 & No & P. aeruginosa & $\mathrm{Si}$ & 45 & 22 & 93-94 & 44 & 24 & 121 & 315 & 57 \\
\hline 15 & M & 22,8 & 75 & $\mathrm{Si}$ & S. aureus & $\mathrm{Si}$ & 70 & 22 & $95-97$ & 127 & 142 & 128 & 127 & 22 \\
\hline 19 & M & 19,3 & 10 & $\mathrm{Si}$ & Flora habitual & No & 60 & 25 & 95-98 & 77 & 52 & 108 & 177 & 33 \\
\hline 20 & M & 20,8 & 25 & $\mathrm{Si}$ & P. aeruginosa & $\mathrm{Si}$ & 65 & 22 & 93-94 & 74 & 42 & 119 & 175 & 29 \\
\hline
\end{tabular}

IMC: Índice de masa corporal; IMCp: Percentil del IMC; Puntaje clínico de FQ según Schwachman: leve (75-100), moderado (40-74) y grave $(<40)$; según Brasfield: leve (20-25), moderado (10-19) y grave $(<9)$, TAC: Tomografia axial computada de tórax. 
Tabla 2. Valores espirométricos y pletismográficos obtenidos en 10 pacientes con FQ. Nótese el promedio obtenido en relación a los valores de referencia

\begin{tabular}{lrrrr}
\hline & m & \multicolumn{1}{c}{$\begin{array}{c}\text { FQ } \\
\text { (rango) }\end{array}$} & $\begin{array}{r}\text { Valor de referencia* } \\
\text { m }\end{array}$ & \multicolumn{1}{c}{ (rango) } \\
\hline $\mathrm{CV}(\mathrm{mL})$ & 2.920 & $(1.730-4.970)$ & 2.960 & $(1.800-5.420)$ \\
$\mathrm{VEF}_{1}(\mathrm{~mL})$ & 2.060 & $(1.350-4.130)$ & 2.600 & $(1.580-4.570)$ \\
$\mathrm{FEF}_{25-75 \%}(\mathrm{~mL} / \mathrm{s})$ & 1.800 & $(880-5.180)$ & 3.075 & $(2.030-4.930)$ \\
$\mathrm{CPT}(\mathrm{mL})$ & 5.620 & $(2.730-7.570)$ & 4.100 & $(2.500-6.640)$ \\
$\mathrm{CRF}(\mathrm{mL})$ & 3.030 & $(1.360-5.390)$ & 2.015 & $(1.220-3.140)$ \\
$\mathrm{VR}(\mathrm{mL})$ & 2.200 & $(940-4.590)$ & 960 & $(660-1.330)$ \\
\hline
\end{tabular}

FQ: Fibrosis quística; m: mediana; $\mathrm{CV}$ : capacidad vital; $\mathrm{VEF}_{1}$ : Volumen espiratorio en el primer segundo; $\mathrm{FEF}_{25-75 \%}$ : Flujo espiratorio forzado en el primer segundo; CPT: Capacidad pulmonar total; CRF: Capacidad residual funcional; VR: Volumen residual. *: Valores de referencia según Knudson (espirometría) y Zapletal (pletismografía).

Tabla 3. Valores pletismográficos obtenidos en 10 pacientes FQ y 33 controles sanos. Nótese el promedio de CPT, VR, VR/CPT y CRF significativamente superior respecto al grupo control $(\mathbf{p}<\mathbf{0 , 0 5}) *$

\begin{tabular}{|c|c|c|c|c|}
\hline & m & $\begin{array}{l}\text { FQ } \\
\text { (rango) }\end{array}$ & & $\begin{array}{l}\text { troles sanos } \\
\text { (rango) }\end{array}$ \\
\hline $\mathrm{CV}(\mathrm{mL})$ & 2.920 & $(1.730-4.970)$ & 3.305 & $(1.830-5.960)$ \\
\hline CPT (mL)* & 5.620 & $(2.730-7.570)$ & 4.540 & $(2.420-8.580)$ \\
\hline $\mathrm{VR}(\mathrm{mL}) *$ & 2.200 & $(940-4.590)$ & 1.325 & (590-2.620) \\
\hline $\mathrm{CRF}(\mathrm{mL})^{*}$ & 3.030 & $(1.360-5.390)$ & 2.260 & $(1.280-3.400)$ \\
\hline VR/CPT (\%)* & 38,5 & (22-61) & 28 & $(20-43)$ \\
\hline
\end{tabular}

FQ: Fibrosis quística; m: mediana; CV: capacidad vital; CPT: Capacidad pulmonar total; CRF: Capacidad residual funcional; VR: Volumen residual.

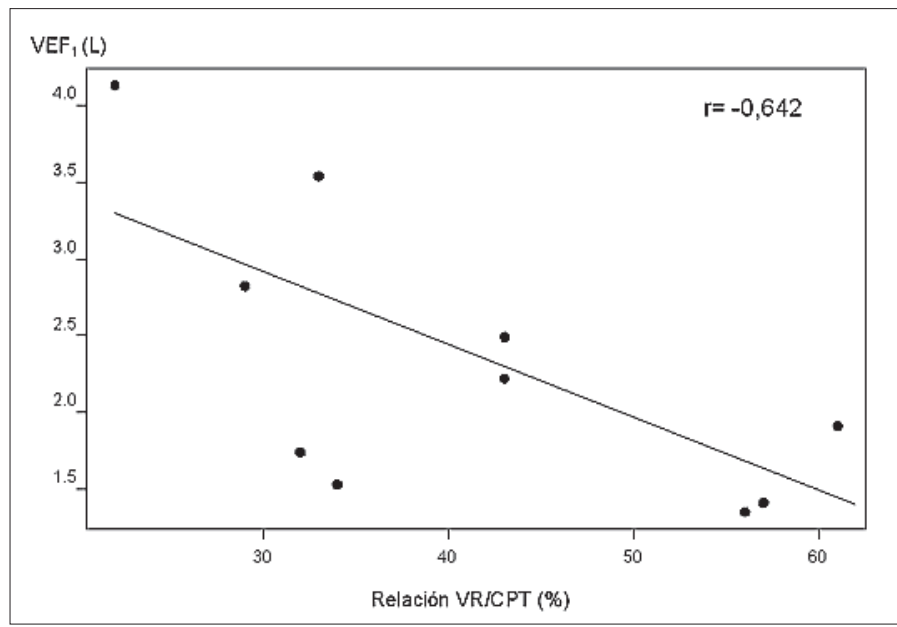

Figura 1. Valores de $\mathrm{VEF}_{1}$ vs relación VR/CPT en pacientes con FQ. Se observa una relación inversamente proporcional entre $\mathrm{VEF}_{1}$ y la relación VR/CPT en los pacientes con FQ ( $r=-0,642 ; \mathrm{p}<0,05)$.
$\mathrm{VEF}_{1}$ disminuido, uno de los cuales fue el que presentó respuesta a salbutamol. En 8 pacientes la relación VR/CPT fue $>30 \%$ (3 de ellos $>50 \%$ ); en $5 / 8$ se diagnosticó atrapamiento aéreo sin cambios al administrar salbutamol. Seis tenían alteración de CRF y VR/CPT (3 con VR/CPT > 50\%). Finalmente, 1/9 mostró obstrucción severa $\left(\mathrm{VEF}_{1}=\right.$ $44 \%$ ) con VR/CPT > 50\%, sin respuesta a broncodilatador, presentando además compromiso nutricional (Tabla 1).

La Tabla 2 muestra los valores espirométricos y pletismográficos observados y los teóricos de referencia según Knudson y Zapletal respectivamente; observándose la disminución del $\mathrm{VEF}_{1}, \mathrm{FEF}_{25-75 \%} \mathrm{y}$ el aumento de CPT, VR y CRF. Al analizar y comparar los resultados obtenidos, se observó que el promedio de CPT y VR fue significativamente superior en los pacientes con FQ respecto a los del grupo control de niños sanos chilenos: 5,62 vs 4,31 L y 2,2 vs $1,2 \mathrm{~L}$ respectivamente $(\mathrm{p}<$ $0,05)$, así como también para VR/ CPT y CRF (Tabla 3), lo mismo se encontró en relación a los valores de referencia. Se observó una relación matemática, inversamente proporcional entre $\mathrm{VEF}_{1}$ y la relación VR/CPT en los pacientes con FQ ( $\mathrm{r}=-0,642 ; \mathrm{p}<0,05) ;$ así como entre el $\mathrm{FEF}_{25-75 \%}$ y la relación VR/ CPT ( $r=-0,803 ; p<0,01)$. Por su parte, los flujos espiratorios revelaron una mayor caída de la pendiente de la curva (relación inversamente proporcional en términos matemáticos) demostrando un mayor compromiso en flujos que en volúmenes (Figuras 1 y 2).

\section{Discusión}

Este estudio descriptivo mostró un incremento significativo de los volúmenes pulmonares en pacientes con FQ aún teniendo espirometría normal. Nuestros hallazgos sugieren una relación matemática inversa entre los flujos pulmonares espiratorios 


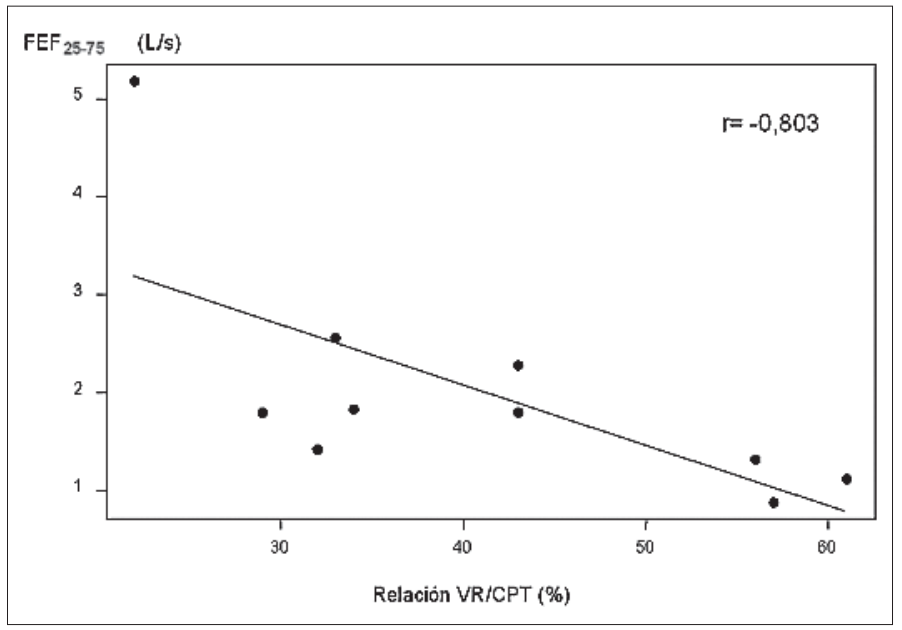

Figura 2. Valores de $\mathrm{FEF}_{25-75 \%}$ vs relación $\mathrm{VR} /$ CPT en pacientes con FQ. Destaca que los flujos espiratorios presentan una mayor caída de la pendiente de la curva que $\mathrm{VEF}_{1}$ observándose un mayor compromiso en flujos que en volúmenes $(r=-0,803$; $p<0,01)$. forzados $\left(\mathrm{VEF}_{1}\right.$ y $\left.\mathrm{FEF}_{25-75 \%}\right)$ y la relación $\mathrm{VR} /$ CPT. Los valores pletismográficos obtenidos en este grupo de 10 pacientes con FQ controlados en nuestro centro, revelaron información importante para el cuidado respiratorio. Nuestros datos demostraron que 9/10 sujetos con FQ presentaban algún grado de atrapamiento aéreo, determinado por PG, incluso uno de ellos no estaba colonizado por microorganismos y tenía espirometría normal.

La PG reveló la presencia de cambios obstructivos de diversos grados. Por otra parte, los volúmenes pulmonares en sujetos con FQ fueron significativamente superiores a los obtenidos en el grupo control o sus respectivos valores de referencia (teóricos) publicados. Se observó una asociación (relación inversa en términos matemáticos) inversamente proporcional entre los flujos espiratorios forzados y los volúmenes pulmonares pletismográficos, lo que es comparable con lo publicado en otras series ${ }^{17}$.

El $V F_{1}$ es el parámetro más utilizado para determinar obstrucción bronquial en cualquier patología pulmonar crónica. Clásicamente el $\mathrm{VEF}_{1}$ ha demostrado ser el instrumento epidemiológico y la herramienta clínica más empleada en la determinación de la progresión de enfermedad respiratoria en niños y adultos con FQ:3,6 sin embargo, como sugiere este estudio, la espirometría fue incapaz de identificar a muchos otros sujetos con limitación obstructiva leve-moderada y compromiso pulmonar como se determinó en la PG. De los 9 pacientes que presentaron alguna alteración en la PG, 2 tenían hiperinsuflación pulmonar (uno con espirometría normal) y otro sólo tenía disminución en los flujos. Esto probablemente se deba a que precozmente exista atrapamiento aéreo antes de los cambios de la espirometría, inclusive del $\mathrm{FEF}_{25-75 \%}$ como fue observado en varios de nuestros pacientes. Por otra parte, en el grupo de pacientes estudiados, la mayoría correspondían a estadío leve a moderado de enfermedad, lo cual podría explicar la escasa alteración espirométrica, observada en una mayor sensibilidad en los flujos espirométricos (7/10 pacientes) y sobre todo en la evaluación de volúmenes pulmonares por $\mathrm{PG}$ en que precozmente determinó compromiso pulmonar. Según lo revisado en forma detallada, no existe experiencia publicada en nuestro medio que determine la relación matemática entre la espirometría y PG en sujetos con FQ.

Varios trabajos han mostrado que la determinación de volúmenes pulmonares es útil para evaluar progresión y respuesta a tratamiento en patologías que pueden evolucionar con volúmenes pulmonares aumentados como es en asma ${ }^{9}$, displasia broncopulmonar ${ }^{10}$ y $F Q$, entre otras. La PG determina de manera óptima la CRF al identificar el atrapamiento aéreo, lo cual se ve limitado con otras pruebas que subestiman el valor de $\mathrm{CRF}^{4,6}$. Esto es de principal importancia en FQ, ya que el deterioro pulmonar es irregular determinando zonas con distinto grado de atrapamiento aéreo, lo que dificulta la medición por gases de la CRF.

Tratando de explicar el compromiso pulmonar precoz en este grupo de enfermos se evalúan diferentes técnicas para determinar atrapamiento aéreo. Se ha determinado el rol temprano de la inflamación, de la infección y el deterioro de la función pulmonar en niños. Farell y colaboradores, mediante el empleo de otras PFP, (técnica oclusión que evalúa mecanismos de respiración 
pasiva, volúmenes pulmonares a través de lavado de nitrógeno y lavado broncoalveolar), encontraron una estrecha relación entre la presencia de inflamación e infección con el mayor grado de atrapamiento aéreo ${ }^{17}$. Lo anterior, lo podemos correlacionar con nuestros pacientes en que incluso en el caso en que no existía la presencia de microorganismos en la vía aérea ya existía compromiso en la pletismografía probablemente secundario a la inflamación. Otros estudios han demostrado la utilidad de la HRCT, la cual tendría la ventaja sobre los métodos de tomografías previas el menor tiempo de duración y grado de exposición a radiación. Long y colaboradores, demostraron que ésta sería capaz de determinar tempranamente en niños con mínimos síntomas cambios de la estructura de la vía aérea secundaria a la inflamación caracterizados por engrosamiento y mayor dilatación en niños con FQ en relación a sus controles ${ }^{8}$.

La mayor limitación de nuestro estudio es el pequeño tamaño de la muestra. Muchos de los pacientes controlados en nuestro centro aún no tienen la capacidad de realizar una PG por su corta edad. Por otra parte, todos los pacientes enrolados presentan puntajes en grado leve (excepto uno, según criterio radiológico) y no severo, por lo que existe un sesgo inherente en la selección de estos 10 casos (según puntaje de Brasfield). Sería ideal ampliar este número de pacientes con FQ especialmente con formas más graves o severas y determinar la relación matemática existente en este subgrupo, trabajo que actualmente está en desarrollo. Finalmente, es importante mencionar que la PG tiene algunas limitaciones: es una técnica que puede llevar a errores en pacientes muy obstructivos. Sus mediciones se determinan a través de ecuaciones que relacionan los cambios de presiones intra y extratorácicas y que permiten calcular el volumen de gas torácico y así la CPT. Por ello, en casos con obstrucción severa puede existir sobreestimación de estos valores ${ }^{18}$, lo que no se observó en nuestros pacientes por su menor grado de severidad, lo cual debe ser considerado en estadios avanzados. La PG en nuestro medio no se encuentra ampliamente disponible por el elevado costo de su implementación.

En resumen, la espirometría es una herramienta útil y complementaria en este grupo de pacientes, su limitación es no poder determinar volúmenes estáticos y pesquisar el compromiso respiratorio obstructivo demostrado por un VR $>130 \%$ en la mayoría de los niños de esta serie. Esto puede ser explicado por un compromiso desigual en los campos pulmonares, por la fisiopatología del atrapamiento aéreo en estos niños y el estadio leve de enfermedad en la mayoría de ellos, lo que le da un mayor rol a la utilización de la PG en FQ. De manera interesante, nuestros hallazgos son similares a los publicados previamente por grupos extranjeros ${ }^{17}$. En conclusión, a pesar del número reducido de pacientes seleccionados y de tratarse de un reporte breve, nuestros hallazgos sugieren que la evaluación con PG fue útil en la evaluación de la FP en pacientes con FQ; sin embargo, se requieren estudios prospectivos que validen nuestros resultados preliminares, que hemos reportado.

\section{Bibliografía}

1.- TIDDENS H A. Detecting early structural lung damage in cystic fibrosis. Pediatr Pulmonol 2002; 34: 22831.

2.- BRODY A S. Early morphologic changes in the lungs of asymptomatic infants and young children with cystic fibrosis. J Pediat 2004; 144: 145-6.

3.- DAVIS P B. The decline and fall of pulmonary function in cystic fibrosis: New models, new lessons J Pediatr 1997; 131: 789-90.

4.- MILNER A D. Lung volume measurements in childhood. Paediatric Respire Rev 2000; 1: 135-40.

5.- MALMBERG L P, PELKONEN A, HAKULINEN A, HERO M, POHJAVUORI M, SKYTTA J, et al. Intraindividual variability of infant whole-body plethysmographic measurements: Effects of age and disease. Pediatr Pulmonol 1999; 28: 356-62.

6.- COREY M, EDWARDS L, LEVISON H, KNOWLES M. Longitudinal analysis of pulmonary function decline in patients with cystic fibrosis. J Pediatr 1997; 131: 809-14.

7.- BRASFIELD D, HICKS G, SOONG S J, TILLER R. The chest roentgenogram in Cystic Fibrosis: A New Scoring System. Pediatrics 1979; 63: 24-9.

8.- $\quad$ LONG F R, WILLIAMS R S, CASTILE R G. Structural airway abnormalities in infants and young children with cystic fibrosis. J Pediat 2004; 144: 154-61.

9.- OLAGUIBEL J M, ÁLVAREZ-PUEBLA M J, ANDA M, GÓMEZ B, GARCÍA B E, TABAR A I, et al. Comparative analysis of the bronchodilator response measured by impulse oscillometry, spirometry and body plethysmography in asthmatic children. J Investig Allergol Clin Immunol 2005; 15: 102-6.

10.- ROBIN B, KIM Y J, HUTH J, KLOCKSIEBEN J, TORRES M, TEPPER R, et al. Pulmonary function in bronchopulmonary dysplasia. Pediatr Pulmonol 2004; 37: 236-42.

11.- KRAEMER R, AEBI C, CASAULTA A, GALLATI S. Early detection of lung disease and its association with the nutritional status, genetic background and life events in patients with cystic fibrosis. Respiration 2000; 67: 477-90.

12.- COATES A L, PESLIN R, RODENSTEIN D, STOCKS J. Measurement of lung volumes by plethysmography. Eur Respir J 1997; 10: 1415-27. 
13.- SHWACHMAN H, KULCZYCKI L. Long-term study of one hundred five patients with cystic fibrosis; studies made over a five- to fourteen-year period. Ama J Dis Child 1958; 96: 6-15.

14.- KNUDSON R J, LEBOWITZ M D, HOLBERG C J, BURROWS B. Changes in the normal maximal expiratory flow-volume curve with growth and aging. Am Rev Respir Dis 1983; 127: 725-34.

15.- ZAPLETAL A, SAMANEK M, PAUL T. Lung function in children and adolescent. Methods, Reference Values. Prog. Resp. Res. 22 Karger, Basel 1987: 83-112.
16.- CAUSSADE S, PULGAR D, DÍAZ C, VIVIANI P, CONTRERAS I, ARANGUIZ C, et al. Volúmenes pulmonares por pletismografía. Valores normales en escolares chilenos (resumen). Rev Chil Pediatr 2004; 75(Supl): S59-S95.

17.- FARRELL P M, LI Z, KOSOROK M R, LAXOVA A, GREEN C, COLLINS J, et al. Longitudinal evaluation of bronchopulmonary disease in children with cystic fibrosis. Pediatr Pulmonol 2003; 36: 230-40.

18.- STANESCU D. Was it just our problem, or yours too? Errors in Body Plethysmography, in infants, children and adults. Pediatr Pulmonol 1991; 11: 285-88.
Correspondencia a:

Dra. Ilse Contreras Estay

Lira 85, $5^{\text {to }}$ piso, Santiago Centro. Casilla postal 114-D

Laboratorio de Respiratorio. Departamento de Pediatría. Pontifica Universidad Católica de Chile.

Fono: 354-3767

Fax: 638-4307

E- mail: ilse@vistamedica.com 